\title{
ON CONGRUENCE INDICES FOR SIMPLE CLOSED CURVES
}

\section{S. G. WAYMENT}

Abstract. L. M. Blumenthal has defined the concept of congruence indices for point sets in his book Theory and applications of distance geometry, Clarendon Press, Oxford, 1953. Blumenthal shows that the circle has congruence indices $(3,1)$ and asks if this characterizes the circle among the class of simple closed curves. In this paper it is established that various classes of simple closed curves do not have congruence indices $(3, n)$ for any $n$. Included in these classes are the polygons, simple closed curves with convex interiors and a straight line segment contained in the curve, and simple closed curves with continuous nonconstant radius of curvature on some arc. Thus any noncircular simple closed curve with congruence indices $(3,1)$ must be very pathological. It is shown that if a simple closed curve has positive planar Lebesgue measure, then it fails to have congruence indices $(3, n)$ for any $n$.

Introduction. Let $K$ be a point set in the plane. A point set $H$ will be said to have property $P(3, K)$ provided that each set of 3 points from $H$ is isometric to some set of 3 points in $K$. The set $K$ is said to have congruence indices $(3, n)$ provided each set $H$ with property $P(3, K)$ and containing more than $3+n$ points is isometric to a subset of $K[1]$.

Let $K$ be the unit circle and let $H$ be the four points consisting of the center of $K$ and the vertices of an equilateral triangle inscribed by $K$. It is easily seen that each three points of $H$ are contained on a circle of radius one, but $H$ is not isometric to four points in $K$. Hence the circle does not have congruence indices $(3,0)$. It is known that the circle has congruence indices $(3,1)$ and the question is immediately raised [1]: If $J$ is a simple closed curve in the plane with congruence indices $(3,1)$, then is $J$ a circle? It is known [1] that the triangle and the rectangle do not have congruence indices $(3,1)$.

In this paper we shall establish that certain classes $C, D$, and $E$ of simple closed curves do not have congruence indices $(3, n)$ for any $n$. The classes $C$ and $E$ contain the regular polygons.

The class $C$. We first describe a rather peculiar property for three points $\left\{x_{0}, y_{0}, z_{0}\right\}$ on a simple closed curve $J$ with convex interior. Let

Received by the editors May 8, 1970.

AMS 1969 subject classifications. Primary 5250.

Key words and phrases. Congruence indices, simple closed curves, lift off property, locally slide a curve about a chord, positive Lebesgue measure. 
$\mathrm{Ch}\left(x_{0} z_{0}\right)$ denote the straight line segment joining $x_{0}$ to $z_{0}$. If it is possible to find continuous functions $x(s)$ and $z(s)$ such that $x(s)$ and $z(s)$ are points of $J$ for each $s$ and $x(0)=x_{0}, z(0)=z_{0}$ and the distance $\rho(x(s), z(s))=\rho\left(x_{0}, z_{0}\right)$ for each $s$ in some interval $[0, l]$, then we shall say that one can locally slide the curve $J$ about the chord $\mathrm{Ch}\left(x_{0} z_{0}\right)$. Let us now define a third function $y(s)$ so that $y(0)=y_{0}$ and the triangle with vertices $\{x(s), y(s), z(s)\}$ is congruent to the triangle with vertices $\left\{x_{0}, y_{0}, z_{0}\right\}$ for each $s$ in $[0, l]$. Suppose that we can locally slide the curve $J$ about the chord $\operatorname{Ch}\left(x_{0} z_{0}\right)$ and suppose that there does not exist $l$ such that $y(s)$ remains on $J$ for all $s$ in $[0, l]$. We shall define this property by saying that the simple closed curve $J$ experiences lift off at $y_{0}$ as we locally slide $J$ about the chord $\operatorname{Ch}\left(x_{0} z_{0}\right)$. We note that one can locally slide the circle about each chord $\operatorname{Ch}\left(x_{0} z_{0}\right)$ and that at no point $y_{0}$ does the circle experience lift off as we locally slide the circle about the chord $\mathrm{Ch}\left(x_{0} z_{0}\right)$. It would be interesting to know if this gives a characterization of the circle. It would also be interesting to know if for each simple closed curve $J$ which is not a circle and for which there exists a chord $\mathrm{Ch}\left(x_{0} y_{0}\right)$ such that we can locally slide $J$ about $\mathrm{Ch}\left(x_{0} z_{0}\right)$, does there then exist a point $y_{0}$ on $J$ such that $J$ experiences lift off at $y_{0}$ ?

The class $C$ is the set of all simple closed curves $J$ satisfying the following three properties:

(1) The interior of $J$ is convex.

(2) There exists a (maximal) straight line segment $\mathrm{Ch}(a b)$ on $J$.

(3) Let $J^{\prime}$ be the reflection of $J$ in the line containing $\mathrm{Ch}(a b)$. Then there exists a simple closed curve $J^{\prime \prime}$ isometric to $J$ such that

(i) $b \subset$ int $J^{\prime \prime}$,

(ii) $J^{\prime \prime}$ intersects $J$ at $x_{0}$ and $y_{0}$,

(iii) $J^{\prime \prime}$ in tersects $J^{\prime}$ at $y_{0}$ and $z_{0}$,

(iv) $y_{0}$ is on the interior of the segment $\mathrm{Ch}(a b)$ and

(v) we can slide the simple closed curve $J^{\prime \prime}$ about $\mathrm{Ch}\left(x_{0} z_{0}\right)$ and $J^{\prime \prime}$ experiences lift off at $y_{0}$.

The following diagram is perhaps helpful in understanding these concepts. We let $w$ be the 'center' of $J^{\prime \prime}$ which is bounded by a circular arc and a straight line segment $\mathrm{Ch}(a b)$.

As we rotate $J^{\prime \prime}$ about $w$, we locally slide $J^{\prime \prime}$ about $\mathrm{Ch}\left(x_{0} z_{0}\right)$ and $J^{\prime \prime}$ experiences lift off at $y_{0}$. We note that an interval $\left[y_{0}, y_{0}^{\prime}\right]$ is 'swept out' by crossings of $\mathrm{Ch}(a b)$ with the various positionings of $J^{\prime \prime}$.

THEOREM 1. No element $J$ from $C$ has congruence indices $(3, n)$ for any $n$.

Proof. Refer to Figure 1 and choose the point set $H$ to consist of 


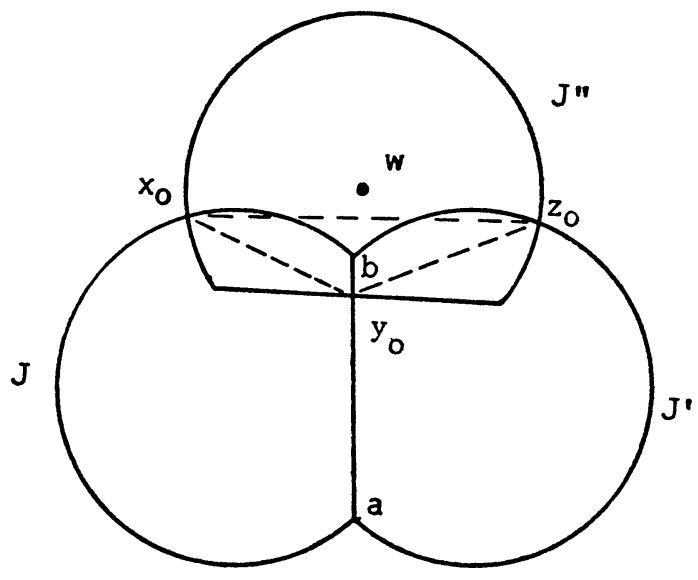

Figure 1

$x_{0}, z_{0}$ and the interval $\left[y_{0}, y_{0}^{\prime}\right]$. If some three points $\left\{p_{1}, p_{2}, p_{3}\right\}$ from $H$ do not include $x_{0}$, then $J^{\prime}$ covers $\left\{p_{1}, p_{2}, p_{3}\right\}$. Similarly if $\left\{p_{1}, p_{2}, p_{3}\right\}$ does not include $z_{0}$, then $J$ covers $\left\{p_{1}, p_{2}, p_{3}\right\}$. If $\left\{p_{1}, p_{2}, p_{3}\right\}$ contains $x_{0}$ and $z_{0}$, then only one $p_{i}$ is from $\left[y_{0}, y_{0}^{\prime}\right]$ and some positioning of $J^{\prime \prime}$ obtained by locally sliding about $\mathrm{Ch}\left(x_{0} z_{0}\right)$ contains $\left\{p_{1}, p_{2}, p_{3}\right\}$. Hence $H$ has property $P(3, J)$ and contains more than $3+n$ points.

The set $H$ is not isometric to a subset of $J$ since if $J_{1}$ is isometric to $J$ (and hence has convex interior) and contains $x_{0}$ and the interval $\left[y_{0}, y_{0}^{\prime}\right]$, then $z_{0}$ is exterior to $J_{1}$ and if $J_{1}$ contains $z_{0}$ and the interval $\left[y_{0}, y_{0}^{\prime}\right]$, then $x_{0}$ is exterior to $J_{1}$. Hence $J$ does not have congruence indices $(3, n)$ for any $n$.

COROLlaRY 1. If $J$ is a regular polygon, then $J$ does not have congruence indices $(3, n)$ for any $n$.

A regular polygon of $n$ sides has been shown $[1, \mathrm{p} .153]$ to have congruence indices $(2 n+1,0)$.

We note also that the boundary of the convex set determined by a circle and a point nearby is in $C$, and we have previously observed (Figure 1) that a circular arc and a straight line segment form a simple closed curve in $C$. Thus the curves which in some sense are most like circles, do not have congruence indices $(3, n)$ for any $n$. The ellipse is also much like a circle and is not contained in $C$.

ThEOREM 2. The ellipse $J_{0}$ does not have congruence indices $(3, n)$ for any $n$.

Proof. We shall outline the proof by referring to Figure 2. 


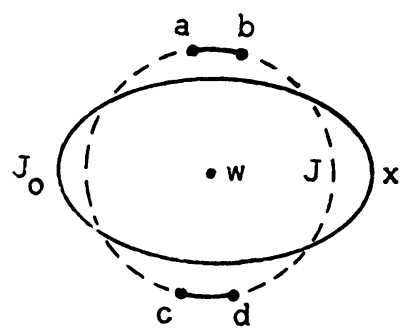

Figure 2

Let $J$ be a circle with radius $r$ which is larger than the largest inscribed circle and smaller than the smallest circumscribing circle. Choose arcs $a b$ and $c d$ on $J$ so that $J \cap J_{0}$ consists of four points and $a b$ and $c d$ are exterior to $J_{0}$, and such that the angles subtended at $w$ by $a b$ and $c d$ are smaller than the angles subtended by the arcs of $J_{0}$ on which the curvature is less than and greater than $1 / r$ respectively.

Case 1. If $\left\{p_{1}, p_{2}, p_{3}\right\}$ are located with $p_{1}$ and $p_{2}$ in $a b$ and $p_{3}$ in $c d$ then rotate and translate $J$ downward until $p_{1}$ and $p_{2}$ are on $J_{0}$. Observe that $p_{3}$ is exterior to $J_{0}$. Now slide the curve $J_{0}$ about the chord $\mathrm{Ch}\left(p_{1} p_{2}\right)$ until $x$ has passed one, but not both of $p_{1}$ and $p_{2}$. If $a b$ has been chosen sufficiently small, then $p_{3}$ will be interior to $J_{0}$. Since the point $p_{3}$ moves continuously from the exterior of $J_{0}$ into the interior of $J_{0}$, there exists a positioning of $J_{0}$ such that $J_{0}$ covers $\left\{p_{1}, p_{2}, p_{3}\right\}$.

Case 2. If $\left\{p_{1}, p_{2}, p_{3}\right\}$ are located with $p_{1}, p_{2}$ and $p_{3}$ in $a b$ and $p_{1}<p_{2}<p_{3}$ with the ordering from $a$ to $b$, then rotate and translate $J$ downward until $p_{2}$ and $p_{3}$ are on $J_{0}$. Since the curvature of $J_{0}$ is less than the curvature of $J$ on the respective arcs subtended by the angle at $w$ determined by $a$ and $b$, we conclude that $p_{3}$ is interior to $J_{0}$. Now slide the curve $J_{0}$ about the chord $\mathrm{Ch}\left(p_{1} p_{2}\right)$ until $p_{2}$ but not $p_{1}$ has passed $x$. If $a b$ has been chosen sufficiently small, then $p_{3}$ will be exterior to $J_{0}$ since the curvature of $J_{0}$ is greater than the curvature of $J$ on the respective arcs subtended by the angle at $w$ determined by $a$ and $b$ relative to the new positioning of $J_{0}$.

Hence $H=a b \cup c d$ has property $P\left(3, J_{0}\right)$ but clearly $J_{0}$ cannot cover $H$ since an ellipse and a circle can intersect in at most four points.

We remark that L. M. Blumenthal (private communication) has established the stronger result that any ellipse $E$ can cover each three points of the circle circumscribing the unique equilateral triangle inscribed in $E$ that has one vertex at an extremity of the major axis of $E$. This result solves for the ellipse the problem posed in the second remark in $[1$, p. 156]. It also follows at once from this that the ellipse does not have congruence indices $(3, n)$ for any $n$. 
We remark further that since nondegenerate hyperbolas and parabolas have continuous curvature and do not contain an arc of any circle, then an argument similar to the one used in the previous theorem for Case 2 with $\left\{p_{1}, p_{2}, p_{3}\right\}$ contained on $a b$ establishes the following result.

THEOREM 3. The nondegenerate parabola and hyperbola do not have congruence indices $(3, n)$ for any $n$.

It is shown in [1, p. 154] that the ellipse and hyperbola have congruence indices $(6,0)$. In [2], J. J. Seidel and J. van Vollenhoven indicate that they have obtained the result that the ellipse and the hyperbola have congruence indices $(5,0)$. L. M. Blumenthal has obtained (unpublished result) that the conics have congruence indices $(5,2)$. We have shown that they do not have congruence indices $(3, n)$ for any $n$. It would be interesting to resolve whether they have congruence indices $(4, n)$ for some $n$.

We remark further that the argument used in Case 2 of the proof of Theorem 2 is extendable to a large class of simple closed curves which we next consider.

The class $D$. Let $D$ be the set of all simple closed curves $J$ in the plane which have the property that $J$ contains an arc $a b$ such that the curvature $k(p)$ is a continuous nonconstant function as $p$ moves from $a$ to $b$.

THEOREM 4. If $J \in D$ then $J$ does not have congruence indices $(3, n)$ for any $n$.

Proof. Since the interval $[0,1]$ does not contain uncountably many disjoint open intervals, it follows that for the simple closed curve $J$ there do not exist uncountably many numbers $r$ such that $J$ contains arcs of circles with radius $r$. Thus, if the curvature $k$ at two interior points $a^{\prime}$ and $b^{\prime}$ of $a b$ satisfy $0<k\left(a^{\prime}\right)=\alpha<k\left(b^{\prime}\right)=\beta$ and $k$ does not assume the value 0 between $a^{\prime}$ and $b^{\prime}$, then there exists a circular arc $c d$ with curvature $k^{\prime}$ between $\alpha$ and $\beta$ and such that $J$ does not contain any circular arc with curvature $k^{\prime}$. Let $p_{1}, p_{2}$, and $p_{3}$ be three points from $c d$ and suppose $p_{1}<p_{2}<p_{3}$ with the ordering from $c$ to $d$. If $c d$ is chosen sufficiently small in arc length, then positioning $c d$ near $a^{\prime}$ with $p_{2}$ and $p_{3}$ on $a b$ will insure $c d$ does not cross $J$ at any other point, and $p$ will be on the (local) side of $a b$ opposite to the (local) side of $a b$ which contains the center of curvature $c_{0}$ for the point $a^{\prime}$ (see Figure 3).

If we slide $J$ about the chord $\mathrm{Ch}\left(p_{2} p_{3}\right)$ until $b^{\prime}$ is between $p_{2}$ and $p_{3}$, 


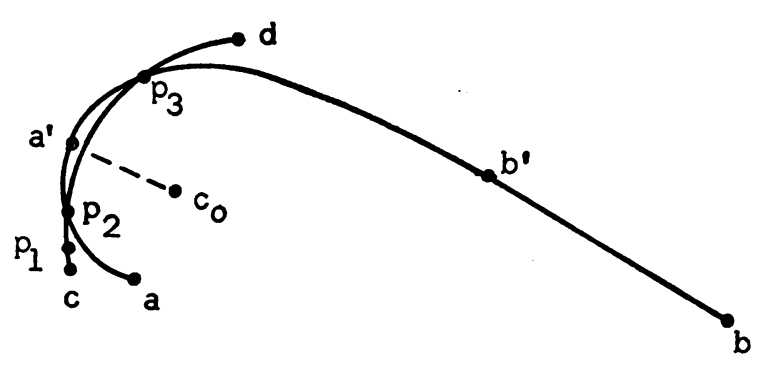

FIGURE 3

then $p_{1}$ is on the same (local) side of $a b$ as $c_{0}$. Consequently there must exist some positioning of $J$ obtained in the sliding process for which $p_{1}, p_{2}$, and $p_{3}$ are on $a b$. Thus $c d$ has property $P(3, J)$ but cannot be isometrically imbedded in $J$.

Missing from $C \cup D$ are the simple closed curves composed of a countable union of straight line segments and a countable union of circular arcs. We shall define a wedge to be a point set consisting of two arcs $a b$ and $b c$ such that $a b \cap b c=b$ and $a b$ is either a straight line segment or a circular arc and $b c$ is either a straight line segment or a circular arc. We further require that the angle at $b$ be less than $180^{\circ}$. In the case that $a b$ or $b c$ is an arc of a circle, a slight modification of the argument in the proof of the following theorem will allow the same conclusion when the angle at $b$ is $180^{\circ}$.

The class $E$. Let $E$ be the class of all simple closed curves $J$ in the plane which have the property that $J$ contains a wedge.

THEOREM 5. If $J \in E$ then $J$ does not have congruence indices $(3, n)$ for any $n$.

Proof. The simple closed curve $J$ does not contain uncountably many disjoint wedges. Let us assume that wedge $W=a b \cup b c \subset J$ and that the smaller of the angles determined by $W$ is $\theta$. Assume for the sake of a picture that $a b$ and $b c$ are straight line segments (see Figure 4).

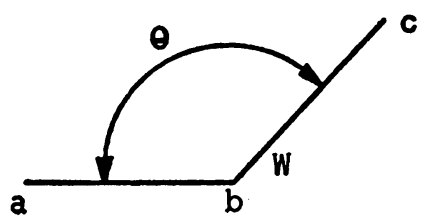

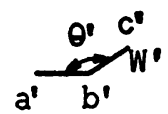

FIGURE 4

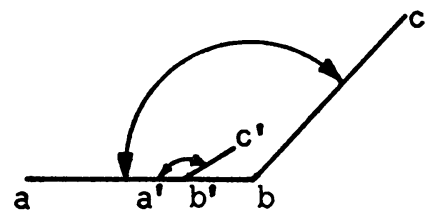

a 
There exists a wedge $W^{\prime}=a^{\prime} b^{\prime} \cup b^{\prime} c^{\prime}$ such that $180^{\circ}>\theta^{\prime}>\theta$ and $J$ contains no wedge with angle $\theta^{\prime}$. By sufficiently restricting the lengths of $a^{\prime} b^{\prime}$ and $b^{\prime} c^{\prime}$ we can insure that any three points $\left\{p_{1}, p_{2}, p_{3}\right\}$ of $W^{\prime}$ can be covered by $W$ by positioning $W^{\prime}$ so that $a^{\prime} b^{\prime} \subset a b$ or $b^{\prime} c^{\prime} \subset b c$ and sliding if necessary. Hence $J$ does not have congruence indices $(3, n)$.

The argument of Theorem 5 can be extended to various other special classes of simple closed curves. We note also that the class $E$ contains the regular polygons (in fact all polygons).

The sets $D$ and $E$ are dense in the set of all simple closed curves under various metrics. Since in these metrics a sequence of polygonal simple closed curves converge to a circle, the density is not helpful in resolving the general question. It is apparent however that if there exists a simple closed curve $J$ which is not a circle and has congruence indices $(3,1)$ then $J$ is quite pathological since $J \notin C \cup D \cup E$. We next consider a pathological class of simple closed curves.

THEOREM 6. If $J$ is a simple closed curve in the plane with positive planar Lebesgue measure, then $J$ does not have congruence indices $(k, n)$ for any $k$ or $n$.

We shall give a series of lemmas before returning to the proof of Theorem 6.

Lemma 1. If $J$ is a set with positive planar Lebesgue measure $\mu(J)$ and $\epsilon>0$, then there exists a closed square disk $D$ such that $\mu(J \cap D) / \mu(D)$ $>1-\epsilon$.

Proof of Lemma 1. Without loss of generality we shall assume $\mu(J)<\infty$. There exists an open set $O$ containing $J$ such that $\mu(O \sim J)$ $<\epsilon \mu(J)$. There exists a disjoint sequence of closed square disks $\left\{D_{i}\right\}$ such that $\mu\left(O-\bigcup_{i=1}^{\infty}\right.$ int $\left.D_{i}\right)=0$. If for each $D_{i}$ we have $\mu\left(J \cap D_{i}\right) / \mu\left(D_{i}\right)$ $<1-\epsilon$, then $\left(\sum \mu\left(J \cap D_{i}\right) / \mu(O)\right)<1-\epsilon$ and hence there must exist $i$ such that $D=D_{i}$ satisfies Lemma 1.

Lemma 2. If $M$ is a Lebesgue measurable subset of the unit interval on the real number line and if for each $t \in[0,1 / 3)$, the triple $\{t, t+1 / 3, t+2 / 3\}$ is not a subset of $M$, then $\mu(M) \leqq 2 / 3$.

Proof of Lemma 2. Let $M_{1}=[0,1 / 3) \cap M, M_{2}=[1 / 3,2 / 3) \cap M$ and $M_{3}=[2 / 3,1) \cap M$. Let $H_{1}=\left\{t \mid t \in[0,1 / 3)\right.$ and $\left.t \notin M_{1}\right\}, H_{2}$ $=\left\{t \mid t \in[0,1 / 3), t \in M_{1}\right.$, and $\left.t+1 / 3 \notin M_{2}\right\}$, and let $H_{3}=\{t \mid t \in[0,1 / 3)$, $t \in M_{1}, t+1 / 3 \in M_{2}$, and $\left.t+2 / 3 \notin M_{3}\right\}$. The $H_{i}$ are pairwise disjoint, measurable, and $\sum_{i=1}^{3} H_{i}=[0,1 / 3)$. Hence $\mu\left(\sum_{i=1}^{3} H_{i}\right)=1 / 3$. Since $H_{1} \cup\left(H_{2}+1 / 3\right) \cup\left(H_{3}+2 / 3\right) \subset[0,1]-M$, we conclude $\mu(M) \leqq 2 / 3$. 


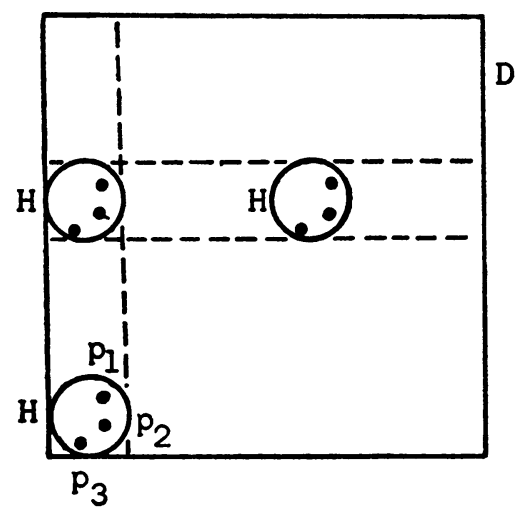

FIGURE 5

LEMмA 3. If $K$ is a Lebesgue measurable subset of the closed unit square disk $D=\{(x, y) \mid 0 \leqq x \leqq 1$ and $0 \leqq y \leqq 1\}$ with $\mu(K)>1-\epsilon^{2}$ for $\epsilon<1 / 3$, then there exists a circular disk $H$ with diameter $\delta<1 / 3-\epsilon$ and such that $H$ has property $P(3, K)$.

Proof of Lemma 3 . Let $\left\{p_{1}, p_{2}, p_{3}\right\}$ be three points in $H$. Translate and rotate $H$ to be tangent to the $x$ and $y$ axes (see Figure 5) and so that no two of the three points $\left\{p_{1}, p_{2}, p_{3}\right\}$ lie on the same horizontal line. Let $Y$ be the set of all $y$ such that the horizontal line segment $l_{y}$ with ordinate $y$ and $0 \leqq x \leqq 1$ intersects $K$ in a set of linear Lebesgue measure less than $1-\epsilon$. Since the planar Lebesgue measure of $D \sim K$ is less than $\epsilon^{2}$, Fubini's theorem assures us that the linear Lebesgue measure of $Y$ is less than $\epsilon$. Since for almost every $y \in[0,1], l_{y} \cap K$ is Lebesgue measurable, by Lemma 2 we can slide $H$ vertically until $p_{1}, p_{2}$, and $p_{3}$ simultaneously lie on horizontal line segments $l_{1}, l_{2}$, and $l_{3}$ with $\mu\left(l_{i} \cap K\right)>1-\epsilon$ for $i=1,2$, and 3 . As we translate $H$ horizontally the points $p_{i}$ sweep out line segments $l_{i}^{\prime}$ of length $1-\delta$ before $H$ becomes tangent to the vertical line $x=1$. Thus the linear measure of $l_{i}^{\prime} \cap K$ is greater than $1-\delta-\epsilon>2 / 3$ for each $i$. Applying Lemma 2 again we can conclude that there exists a positioning of $H$ such that $\left\{p_{1}, p_{2}, p_{3}\right\} \subset K$.

Proof of Theorem 6. It follows immediately from Lemmas 1 and 3 that if $J$ has positive planar measure, then there exists a circular disk with property $P(3, J)$. Since $J$ cannot contain a disk, it follows that $J$ does not have congruence indices $(3, n)$ for any $n$. By appropriately adjusting Lemmas 2 and 3 we can conclude that $J$ does not have congruence indices $(k, n)$ for any $k$ or $n$. 
COROLlaRY. If $K$ is a subset of the plane with positive planar Lebesgue measure and $K$ does not contain an interior point, then $K$ does not have congruence indices $(k, n)$ for any $k$ or $n$.

\section{REFERENCES}

1. L. M. Blumenthal, Theory and applications of distance geometry, Clarendon Press, Oxford, 1953. MR 14, 1009.

2. L. Danzer, B. Grünbaum and V. Klee, Helly's theorem and its relatives, Proc. Sympos. Pure Math., vol. 7, Amer. Math. Soc., Providence, R. I., 1963, p. 139. MR 28 \#524.

Utah State University, Logan, Utah 84321 